protoplasm mass is seen imbedded a nucleus. Osmic acid, safranin; Leitz, ob. 7, oc. 3 . Figs. 2 and 3 , newly formed nerre fibres with nuclei from the central end 36 days after division of the nerve. Osmic
acid, safranin ; Leitz, ob. 7 , oc. 1. In Fig. 4, in the uppermost part of the preparation is seen a spindle-shaped nucleus with detached process. Below this is seen a degenerated nerve fibre with balls of mreline and farther down spindle-shaped nuclei with protoplasm processes. The peripheral part seven days after division of the nerve. Osmic acid, safranin ; Leitz, ob. 7, oc. 3. Fig. 5,
newly formed nerve fibres with nuelei from the peripheral part. newly formed nerve fibres with nuelei from the peripheral part. 108/1. Fig. 6, newly formed fibre being detached from an old fibre. Its nucleus has re-divided. Tenth day, van Giesen; Leitz, ob. 7, oc. 1. Fig. 7, newly formed nerve fibre with spindle-shaped nucleus between two old nerve fibres. On the old fibres are seen two shorter nuclei that are supposed to belong to the sheath of Schwann. Central part, tenth day, van Giesen; Leitz, ob. 7, oc. 2. Fig. 8, from same preparation. The microscope has been focussed upon two nuclei outside the old fibres. Fig. 9, a newly formed nerve fibre with nucleus is seen detached from the tissue full of nuclei near the place of union. The nucleus and the fibre are about to be re-divided. Farther in a central direction on the fibre is seen a piece of myeline and still further central is a nucleus which is not yet divided. From the central part, fortyfifth day, van Giesen; Swift ob. 1/6 in., pr. oc. 2, 332/1. Fig. 10, $a$ and $b$, two bundles of newly formed fibres from the central end of a nerve in the neighbourhood of the place of union. Between the ends of them are seen a single detached nucleus with a fine slender nerve thread. Behind and to the side of them is seen a connective tissue fifth day, van Giesen; Swift, ob. $\frac{1}{2}$ inch, pr. oc. 2 , cn. 113/1. Plate $V$. Fig. 11, in the lower part of the figure is seen a nerve fibre of normal thickness. At first it is darkly stained and of granular appearance, then a newly formed fibre as a black streak along the middle of the old fibre is seen suddenly. The old fibre is torn on the other side of Ranvier's node +, after which the young fibre is seen detached and broken farther in a central direction ++ . On the other side of Ranvier's node is seen another piece of newly formed fibre. Fig. 12, detached fibres in different stages of development near the place of union. Central piece, sixtieth day, formalin, Weigert's stain ; Swift, ob. $\frac{1}{2}$ in., pr. oc. 2, cn. 120/1. Fig. 13, nerve fibres with short segments and spindle-shaped thickenings. From the connective tissue near the place of union. Seventy-seventh day, Weigert's myeline sheath stain Swift, ob. $\frac{1}{2}$ in., pr. oc. 2 , cn. $90 / 1$. Fig. 14, newly formed nerve fibres from the peripheral part of the interrupted and not united nerve. Sixtieth day, Weigert's myeline sheath stain; Swift, ob. $1 / 6$ inch, pr. oc. 2 cn. 340/1. Fig. 15, transverse section from the muscle of the interrupted nerve. Sixtieth day, van Giesen; Leitz, ob. 7 , oc. 1 . Fig. 16 transverse section from the muscle of the united nerve. Sixtieth day, van Giesen ; Leitz, ob. 7, oc. 1.

Christiania.

\section{THREE CASES OF LIGATURE OF THE COMMON CAROTID ARTERY.}

By Sir W. J. COLLINS, M.S. Lond., F.R.C.S. EnG., SURGEON TO THE ROYAL EYE HOSPITAL AND TO THE LONDON TEMPERANCE HOSPITAL.

DURING the past year in three cases I have had to tie the common carotid artery at the London Temperance Hospital. The results in the first two cases may justify their publica. tion. I am indebted to Mr. J. S. Hosford, the surgical registrar for memoranda from which I have drawn up the following notes.

CASE 1. Intracranial aneurysm; ligature of the common carotid artery ; recovery. - The patient was a man, aged 54 years, who had suffered from bronchitis and asthma, had taken a good deal of alcohol, gave no history of syphilis, but gave an account of having suffered from both hæmoptysis and hæmatemesis some 14 years ago. His present illness began with a fall in the first week of December, 1902, in which he struck the right brow. He vomited but did not lose consciousness. Three days afterwards be began to see double and his wife observed that he had a cast in the right eye. He attended at the Royal Eye Hospital, where proptosis of the right eye was observed in the early days of January, 1903. Some chemosis and ecchymosis of the conjunctiva of the right lower eyelid were also noticeable and the patient complained of some pain and noise in the head. admitted him into the London Temperance Hospital under my care on Jan. 9th. On admission the patient was a thin, spare man looking more than his age. There was marked proptosis of the right eyeball, and on palpation distinct pulsation was ito be felt around the globe, in the region of the upper lid. A loud systolic bruit was heard over the right temporal, frontal, and superciliary regions. There were chemosis and hæmorrhagic discolouration of the conjunctiva of the lower lid, with some sero-sanguineous discharge, increased by some ectropion of the lid. The right cornea was clear and the globe seemed to be natural, though displaced somewhat forwards and outwards. The media and fundus were normal. Vision was $\frac{6}{8}$ and J. 1, with $+3 \mathrm{D}$. The left eye was normal. The patient complained greatly of the blowing noise within the head which prevented him from sleeping. I diagnosed intracranial aneurysm and decided to tie the right common carotid artery. On Jan. 19th the patient was placed under chloroform. During the struggle while passing under the anæsthetic there was some oozing of blood from the right orbit. I then tied the common carotid with kangaroo tendon, just above the right omo. hyoid muscle. Pulsation of the globe at once ceased and the bruit was no longer audible. On the next day (Jan. 20th) the patient was very comfortable and was greatly relieved by the complete cessation of the noise in the head. The ligation wound healed per primam in a week. On Feb. 6th proptosis was still present and there was considerable ectropion of the right lower eyelid from solid œedema of the conjunctiva. On several occasions a temporary return of the bruit (though never so loud as before) was observed both by the patient himself and with the stethoscope by others. On other days no bruit whatever could be heard on careful auscultation. The patient was kept under close observation during the month of March and no pulsation or bruit was on any single occasion detected. Some ectropion of the lower eyelid still continued. He was discharged on March 31st.

CASE 2. Slloughing and homorrhagic ulceration of the right tonsillar region (? malignant); ligature of the common carotid artery; great amelioration. -The patient, a man, aged 53 years, a labourer, had enjoyed fair health, with the exception of double inguinal hernia, until January, 1901, when he began to suffer from an ulcer on the buccal mucous membrane of the right cheek. There was no history of syphilis. A month later a fulness was observed in the right temporal region. The ulcer and the temporal swelling continued to increase and on May 8th, 1902, he was admitted to the London Temperance Hospital under my care. On admission the patient was rather flabby and cachectic, with rigid arteries and some emphysema. His breath was very foul, he was unable to open his mouth, and a large diffuse swelling occupied the region above and below the right zygoma. A few enlarged glands could be felt in the upper triangles of the neck. So far as the imperfect examination of the mouth permitted a sloughing and bleeding ulcer appeared to occupy the region of the right tonsil and anterior pillar of the fauces extending to the buccal mucous membrane. An incision of the temporal swelling yielded nothing but blood. During the week after his admission he on several occasions had sharp attacks of hæmorrhage from the mouth and also from the incision which had been made in the temporal region. The fotor from the mouth was abominable, the pain was very great, and the patient was fed with great difficulty; moreover, hæmorrhage of a dangerous amount appeared to be imminent. Having diagnosed malignant disease of or around the right tonsil I advised, and on May 15th performed, ligature of the right common carotid artery. The patient took the anæsthetic well and the ligation was effected just above the omo-hyoid muscle. The wound healed per primam. On June 4th the patient was much improved in his general condition, the pain was greatly relieved, and there had been no return of bæmorrhage. The patient was discharged and on August 14th presented himself as an out-patient, looking remarkably well. The swelling had completely disappeared and though there was still some ulceration of the mouth there had been no hæmorrhage and there was no foetor.

CasE 3. Recurrent carcinoma of the tongue, tonsil, and floor of the month; ligature of the common carotid artery. A married woman, aged 37 years, a multipara, was admitted into the London Temperance Hospital under my care with a small ulcerated swelling in the right side of the tongue. The right half of the tongue was excised after ligature of the lingual and some glands were removed from below the jaw in January, 1902. At the time of this latter operation the patient was pregnant. On March 13th, 1902, she was readmitted with a return of undoubted epithelioma of the base of the tongue. On Feb. 18th she had been delivered of a dead foetus. The new growth involved the tonsil and was firmly planted in the tissues around the tonsil and angle of the jaw and did not admit of removal. On March 20th, under chloroform, I ligatured the right common carotid artery immediately above the omo-hyoid muscle, with a view to check the growth. The wound healed per primam and the patient left the hospital on April 13th in less pain and in improved general health, but without any marked change in the size of the tonsillar growth.

Regent's Park, N.W 


\section{NARCOTILE, BICHLORIDE OF METHYL- ETHYLENE : A NEW GENERAL AN ASTHETIC. ${ }^{1}$}

By TOM EASTHAM, M.B., OH.B. VICT.,

RESIDENT MEDICAL OFFICER TO THE ROXAL BOSCOMBE AND WEST HANTS HOSPTYAL.

I WISH to bring before you a few observations and notes made about this new anæsthetic, especially as this is the first time that narcotile has been introduced to any society in this country or on the continent. For many years there has been a great demand for an anæsthetic which would act rapidly and effectually and at the same time produce no troublesome after-effects. To fulfil these requirements several anæsthetics of the ethyl chloride variety have been extensively used with varying degrees of success, but until the introduction of narcotile none of these bodies could be relied upon. Narcotile is stated to be a pure and definite ether obtained by the direct reaction of hydrochloric acid on mixed ethylic and methylic alcohols distilled together. The vapours are condensed under pressure and purified. It is a pure and constant chemical product and is not subject to decomposition when exposed to light. It is a transparent, colourless, mobile, and highly volatile liquid possessing an agreeable odour. Like ether it is inflammable and cannot be used in the neigbbourhood of an artificial light. It is stored in tubes of a capacity of 50 cubic centimetres.

The apparatus for administering narcotile consists of a glass or metal cylinder fitted with a screw-cap mount and provided with inlet and outlet screw valves. The two-

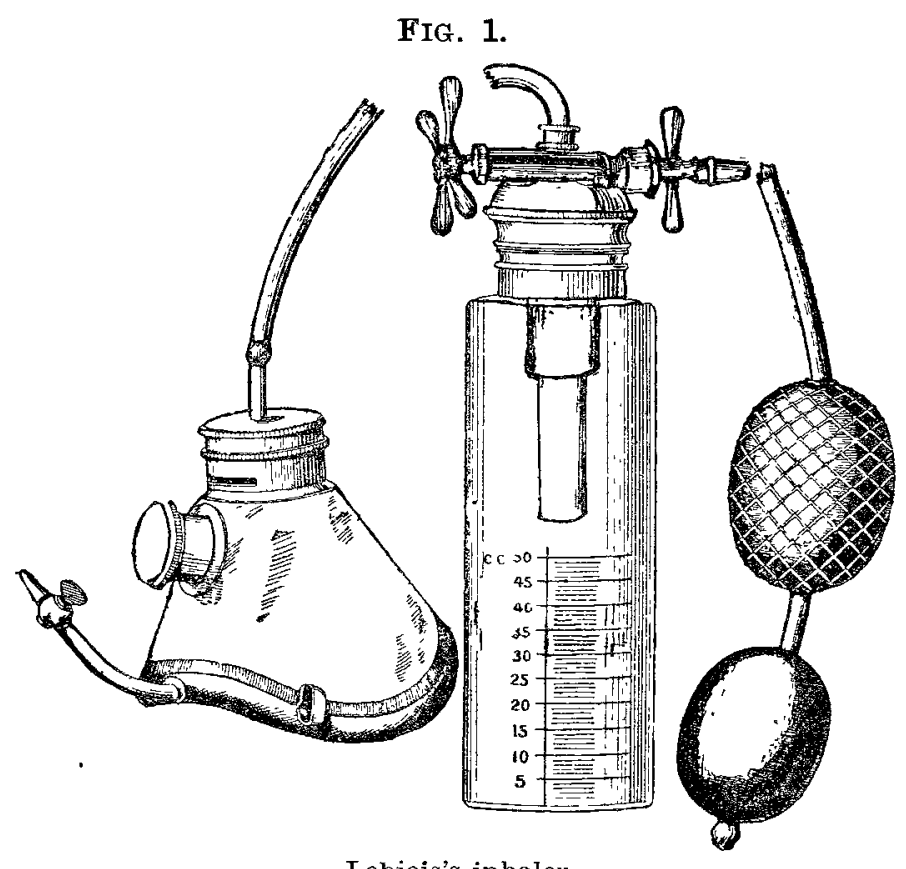

Lobjois's inhaler.

spoked valve communicates with the inlet tube and receives the hand-bellows for pumping air through the bottle. The four spoked valve, situated on the top of the apparatus, connects by means of an india-rubber tube the outlet with a face-piece. The face-piece is made of transparent celluloid and is fitted with an adjustable inlet valve and an automatic expiratory valve. For operations upon the mouth or throat Dr. Doyen's gag fitted with an inhaling tube is extremely useful. The anæsthetic can be given by the nose or by means of a metal tube.

The administration of narcotile. - A space of two hours from the last meal is advisable, but not essential, before undergoing anæsthesia. In my series of cases vomiting has not occurred after a short anæsthesia, but when the patient has had narcotile for several minutes immediately after a meal vomiting has occurred in a few cases. Vomiting caused by this anæsthetic is not very dangerous because the patient is invariably conscious of what is taking place

${ }^{1}$ A paper read before the Bournemouth Medical Society on March 11th, 1903. before the act of vomiting is completed. The patient's neck and waist must be free from all constricting clothing; the patient is placed in any position to suit the operator, preferably the dorsal posture. After widely opening the valves on the bottle the face-piece is held a little distance from the

FIG. 2.

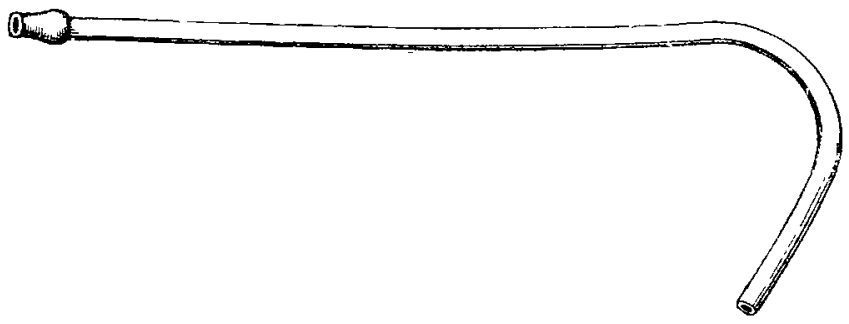

Metal tube.

face so as to accustom the patient to breathe the anæsthetic without discomfort. The face-piece is then carefully fitted to the patient's face and the bellows are pumped at the end of every expiratory movement. The amount of air is regulated by means of an adjustable air valve; very little experience is necessary to tell how much air is required for each patient. After a few inspirations the air slot is usually

FIG. 3.

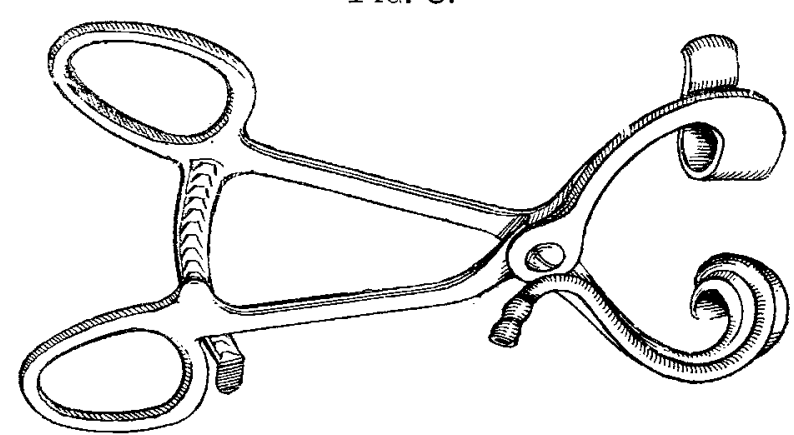

Doyen's gag.

completely closed, so that the patient only breathes air saturated with narcotile vapour. There appears to be little danger of asphyxia or cyanosis on account of the constant insufflation of the bellows, which provides the patient with a plentiful supply of air.

The physiological action.-The general effects of the anæsthesia produced by narcotile resemble greatly those of ether. Like ether it causes a peripheral vascular dilatation, which reveals itself in flushing, redness of the skin, and sweating. The salivary and bronchial secretions are not markedly increased. Respiration is at first quickened and deepened in character; it resembles markedly the noisy breathing of ether anæsthesia. Later the breathing is of the soft snoring or stertorous variety and finally becomes shallow. The heart's action is at first increased in force and rate; later the excitement subsides. Shock and collapse have never supervened. The muscles at first become rigid but soon are completely relaxed. If a limb be raised it falls like an inert mass. When the anæsthesia is complete there is no muscular rigidity. An extremely useful sign of commencing anæsthesia is the loss of the lines and ridges above the nose due to the relaxation of the occipitofrontalis muscle. By thus watching the facial ridges the eye need never be touched before consciousness is lost.

The eye effects.-At first the pupils dilate, soon become smaller, and often markedly contracted. The conjunctival and light reflexes disappear. If more narcotile be given the pupils dilate and the eyelids separate, showing that too much of the anæsthetic is being given.

The anæsthesia is rapid. The recovery is remarkable on account of its extreme rapidity and is usually unaccompanied by headache, giddiness, or other unpleasant symptoms. Soon after the patients "come to" they can express themselves clearly. If the operation is commenced before the patient is anæsthetised the patient is usually in a semi-conscious state and knows what is taking placehears and feels external objects. If the narcotile is increased the patient becomes insensible to pain and yet sees and knows what is going on around him, but if the narcotile is further increased its anæsthetic properties are as efficacious as those of ether or chloroform.

$$
\text { ○ } 3
$$

